\title{
Improved Hybrid Algorithm of Path Planning for Automated Guided Vehicle in Storage System
}

\author{
Jian Wang, Xinhua Zhu*, Minhuan Guo, Surui Yao and Yan Su \\ School of Mechanical Engineering, Nanjing University of Science \& Technology, Nanjing 210094, China \\ ${ }^{*}$ Corresponding author
}

\begin{abstract}
Aiming at the problem of automated guided vehicle path planning in storage system, this paper provided a new method which combines global path planning algorithm and local path planning algorithm to achieve the goal of finding the optimal path. In this paper, the improved $A^{*}$ algorithm is used as the global path planning algorithm, and the improved APF algorithm is used as the local path planning algorithm. The hybrid algorithm not only makes full use of the known information to generate the global optimal path, but also can effectively avoid obstacles on the path. The advantages and effectiveness of the hybrid algorithm are proved by the results of simulations and applications.
\end{abstract}

Keywords-automated guided vehicle; path planning; $A^{*}$ algorithm; APF algorithm; improved hybrid algorithm

\section{INTRODUCTION}

With the rapid development of Internet of things, intelligent storage system is becoming important. In the storage system, the goods are large and the road condition is complicated. In order to quickly find target goods, more and more warehouses began to use the automated guided vehicle [1-2]. Path planning is an important subject in the research of automated guided vehicle related technologies. According to the perception degree of automated guided vehicle to environmental information, path planning is divided into two kind: (1) global path planning with fully known environment information, (2) local path planning with unknown or locally unknown environmental information [3]. In the global path planning, a moving path from the start point to the target point is planned according to the acquired global environment information [4]. This method usually has the disadvantages of large computation, poor real-time performance and can't adapt to the dynamic uncertain environment [5]. The main methods used in global path planning algorithms include A* algorithm, visibility graph method and topological graph method, etc. Local path planning is based on the current local environment information for path planning. The results of local path planning are more real-time and practical, but the local path planning method also has the problem of dropping into local minima or unreachable targets [6]. The main methods used in local path planning algorithms include APF (artificial potential field) method, genetic algorithm and neural network method, etc.

In the storage system, many random factors lead to complicated road environment. Therefore, the automated guided vehicle used in the storage system needs to meet the requirements of safety, low consumption and adaptation to environmental changes. Traditional single path planning algorithms are difficult to meet these performance requirements.
This paper provided a new method which combines global path planning algorithm and local path planning algorithm to achieve the goal of finding the optimal path. In this paper, the improved $\mathrm{A}^{*}$ algorithm is used as the global path planning algorithm, and the improved APF algorithm is used as the local path planning algorithm. The hybrid algorithm not only makes full use of the known information to generate the global optimal path, but also can effectively avoid obstacles on the path.

\section{Design of Global Path PlanNing Algorithm}

Taking into account the time, energy consumption and work efficiency and other factors, the shortest path is chosen as the optimal path when designing the global path planning algorithm. In this paper, the improved $\mathrm{A}^{*}$ algorithm is used to design the global path planning algorithm in the storage system.

\section{A. Storage Environment Map Design}

Path planning consists of two steps: (1) establish an environmental map that contains barrier areas and free areas, (2) choose the appropriate path search algorithm in the environment map. Therefore, the environment map should be built before designing the path planning algorithm. Traditional A* algorithms usually use raster maps. Raster maps are easy to create and maintain, but require huge storage space and computational complexity. In the storage environment, the automated guided vehicle usually only needs to arrive at the fixed several positions, and does not have to traverse all the spaces. Therefore, the grid maps can be changed into topological maps to reduce the amount of computation, and saving the computing time. Topological maps select nodes to represent specific locations on the road, and use the relation between nodes and nodes to represent the links of the roads.

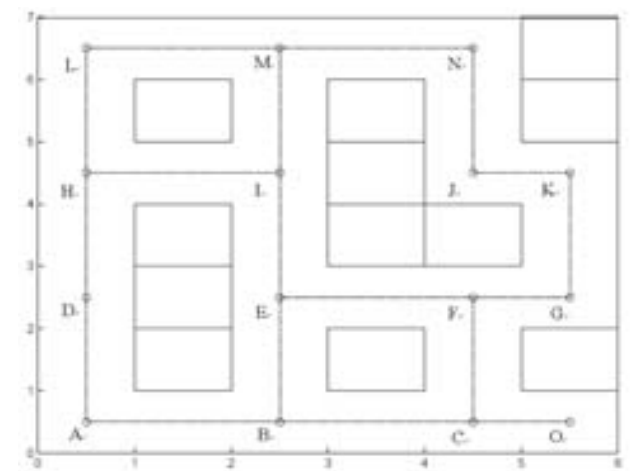

FIGURE I. SKETCH MAP OF STORAGE ENVIRONMENT 
Sketch map of storage environment is depicted in Figure 1. Each inflection point of the environment map is taken as a topological node, and the road connected with the topological node is taken as a topological edge. In the map, set up as shown in Figure 1, A to O, a total of 15 topological nodes. In order to save and search the topological graph, the adjacency matrix is used to represent the relations among nodes. If the topological map has $\mathrm{n}$ topological node: $v_{1}, v_{2}, \ldots, v_{n}$, and the adjacency matrix is a $\mathrm{n} * \mathrm{n}$ matrix. By assigning attributes to each side to distinguish between passable and impassable paths, and the weights of the passable paths are set to 1 , and the weights of the impassable paths are set to 0 . The adjacency matrix is represented as in below:

$$
A_{G}=\left[\begin{array}{lllllllllllllll}
0 & 1 & 0 & 1 & 0 & 0 & 0 & 0 & 0 & 0 & 0 & 0 & 0 & 0 & 0 \\
1 & 0 & 1 & 0 & 1 & 0 & 0 & 0 & 0 & 0 & 0 & 0 & 0 & 0 & 0 \\
0 & 1 & 0 & 0 & 0 & 1 & 0 & 0 & 0 & 0 & 0 & 0 & 0 & 0 & 1 \\
1 & 0 & 0 & 0 & 0 & 0 & 0 & 1 & 0 & 0 & 0 & 0 & 0 & 0 & 0 \\
0 & 1 & 0 & 0 & 0 & 1 & 0 & 0 & 1 & 0 & 0 & 0 & 0 & 0 & 0 \\
0 & 0 & 1 & 0 & 1 & 0 & 1 & 0 & 0 & 0 & 0 & 0 & 0 & 0 & 0 \\
0 & 0 & 0 & 0 & 0 & 1 & 0 & 0 & 0 & 0 & 1 & 0 & 0 & 0 & 0 \\
0 & 0 & 0 & 1 & 0 & 0 & 0 & 0 & 1 & 0 & 0 & 0 & 0 & 0 & 0 \\
0 & 0 & 0 & 0 & 1 & 0 & 0 & 1 & 0 & 0 & 0 & 0 & 0 & 0 & 0 \\
0 & 0 & 0 & 0 & 0 & 0 & 0 & 0 & 0 & 0 & 1 & 0 & 0 & 1 & 0 \\
0 & 0 & 0 & 0 & 0 & 0 & 1 & 0 & 0 & 1 & 0 & 0 & 0 & 0 & 0 \\
0 & 0 & 0 & 0 & 0 & 0 & 0 & 1 & 0 & 0 & 0 & 0 & 1 & 0 & 0 \\
0 & 0 & 0 & 0 & 0 & 0 & 0 & 0 & 1 & 0 & 0 & 1 & 0 & 1 & 0 \\
0 & 0 & 0 & 0 & 0 & 0 & 0 & 0 & 0 & 1 & 0 & 0 & 1 & 0 & 0 \\
0 & 0 & 1 & 0 & 0 & 0 & 0 & 0 & 0 & 0 & 0 & 0 & 0 & 0 & 0
\end{array}\right]
$$

\section{B. Design of A* Algorithm Based on Topological Map}

The kernel of the $A^{*}$ algorithm is to design an evaluation function for each road node, as shown in the following equation:

$$
f(s)=g(s)+h(s)
$$

where,

$f(s)=$ the estimated length from the start node to the goal node, $g(s)=$ the path length from the start node to the current node,

$h(s)=$ the estimated value of the current node to the goal node.

The premise that $A^{*}$ algorithm can search the optimal path is as in below:

$$
h(s) \leq \cos t^{*}\left(s, s_{\text {goal }}\right)
$$

where, $\cos t^{*}\left(s, s_{\text {goal }}\right)=$ the optimal distance from the current node to the goal node. The larger the $h(s)$ value, the fewer the extension nodes. In order to ensure the optimality of the search path, Manhattan distance, diagonal distance or Euclidean distance are used as heuristic functions. In order to guarantee the Equation (3) constant, the Euclidean distance is used as the heuristic function. For a given two coordinate positions $\left(x_{i}\right.$, $\left.y_{i}\right)$ and $\left(x_{j}, y_{j}\right)$, their Euclidean distance is expressed as:

$$
d_{e}=\sqrt{\left(x_{i}-x_{j}\right)^{2}+\left(y_{i}-y_{j}\right)^{2}}
$$

The A* algorithm manage road nodes with two sets of OPEN and CLOSED. OPEN stores sub nodes of extended road nodes which belong to extended nodes. CLOSED stores extended nodes. In the initial case, only the start node is stored in the OPEN, and the CLOSED is empty. In addition, the g values of all nodes are initialized to infinity except for start node.

The flow chart of the A* algorithm is shown in Figure 2. After the algorithm search starts, extend the minimum value of $f$ (s) from the OPEN. The extended child node from $s$ is stored in the OPEN. After the node s extension is complete, move from OPEN to CLOSED. Loop the process until extended to the target node or when OPEN is empty. If OPEN is empty, it indicates no feasible path, and the planning fails. If there is a feasible path, the $\mathrm{A}^{*}$ algorithm must be able to search for it.

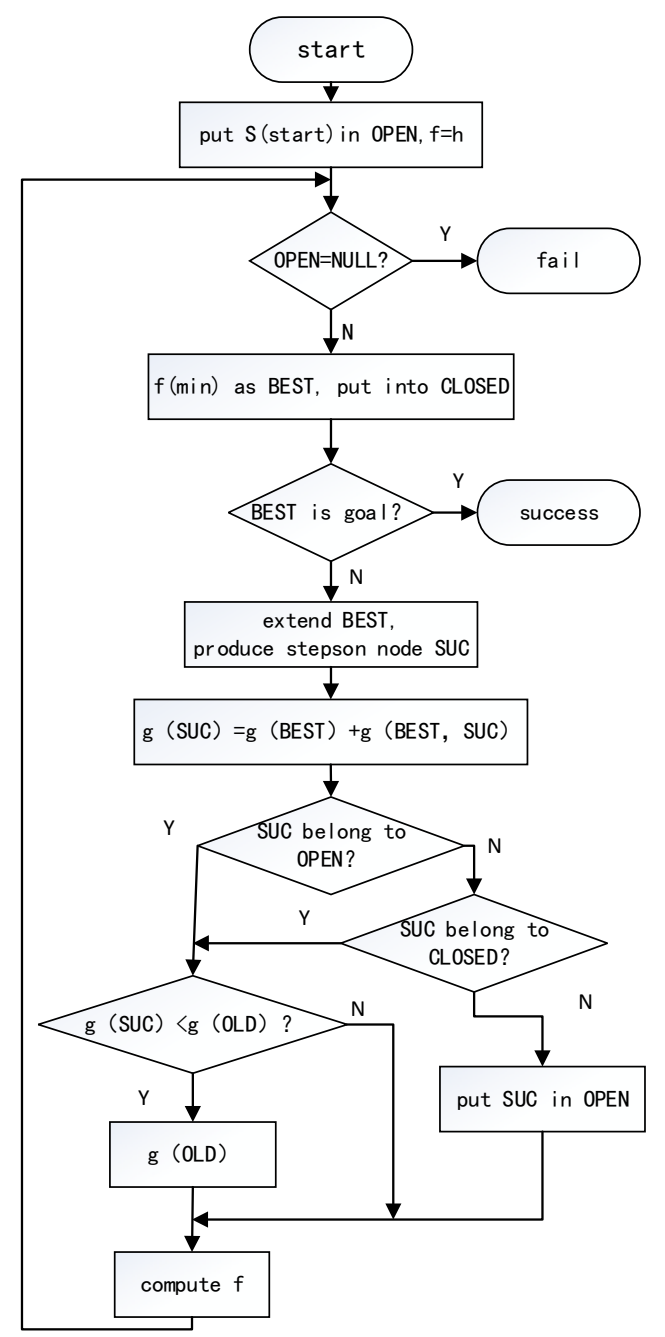

FIGURE II. THE FLOW CHART OF THE A* ALGORITHM 


\section{DESIGN OF LOCAL PATH PLANNING ALGORITHM}

The improved APF (artificial potential field method) is used for local path planning. The APF is first proposed by Khatib. Its essence is to define an abstract potential field of the robot's running space. The potential field is the superposition of the gravitational field in the target position and the repulsive field of the obstacle in the environment [9]. The traditional APF is defined as below.

Assume that the location of the automated guided vehicle is $X=(x, y)$, and the potential field function between the automated guided vehicle and the target point is represented as in below:

$$
U_{a t t}(X)=\frac{1}{2} K_{a t t}\left(X-X_{g}\right)^{2}
$$

where, $K_{a t t}=$ gravitational potential field constant, $X_{g}=$ the position vector of the target point in the earth coordinate system. The gravitational $F_{a t t}(X)$ is defined as the negative gradient of the gravitational field:

$$
F_{a t t}(X)=-\Delta U_{a t t}(X)=-K_{a t t}\left|X-X_{g}\right|
$$

Repulsive potential field function between the automated guided vehicle and the obstacles is represented as in below:

$U_{\text {rep }}(X)=\left\{\begin{array}{cl}\frac{1}{2} K_{\text {rep }}\left[\frac{1}{X-X_{o b s}}-\frac{1}{D}\right]^{2} & , X-X_{o b s} \leq D \\ 0 & , \text { others }\end{array}\right.$

where, $K_{\text {rep }}=$ repulsive potential field constant, $X-X_{o b s}=$ the relative distance between the automated guided vehicle and an obstacle, $D=$ safe distance. The repulsive force $F_{r e p}(X)$ is defined as the negative gradient of the repulsive potential field:

$$
\begin{gathered}
F_{r e p}(X)=-\Delta U_{r e p}(X)= \\
\left\{\begin{array}{cc}
K_{r e p}\left(\frac{1}{X-X_{o b s}}-\frac{1}{D}\right) \frac{1}{\left(X-X_{o b s}\right)^{2}} \frac{\partial\left(X-X_{o b s}\right)}{\partial X}, X-X_{o b s} \leq D \\
0 \quad, \text { others }
\end{array}\right.
\end{gathered}
$$

Therefore, the total potential field and the total force of automated guided vehicles in the storage environment are represented as in below:

$$
\begin{gathered}
U_{\text {total }}(X)=U_{a t t}(X)+U_{\text {rep }}(X) \\
F_{\text {total }}(X)=F_{a t t}(X)+F_{\text {rep }}(X)
\end{gathered}
$$

The main feature of the APF is that the motion direction is determined by the potential field and the gradient direction of the current position. Therefore, compared with other local path planning algorithms, it has the advantages of small computation and real-time performance. But it also has limitations in practical applications, such as when obstacles appear near the target point, the gravity decreases and the repulsion increases, the automated guided vehicles will be hovering near the target when it approaches the target point. This situation is called target unreachable.

For the problem of unreachable target, the method of redefining repulsive force potential function is adopted. The improved repulsive potential field function is as below:

$$
U_{\text {rep }}(X)=\left\{\begin{array}{c}
\frac{1}{2} K_{r e p}\left[\frac{1}{X-X_{o b s}}-\frac{1}{D}\right]^{2}\left(X-X_{g}\right)^{n}, X-X_{o b s} \leq D \\
0 \quad, \text { others }
\end{array}\right.
$$

where, $0<n \leq 1$. The repulsive force of the automated guided vehicle is represented as in below:

$F_{\text {rep }}(X)=-\Delta U_{\text {rep }}(X)=\left\{\begin{array}{cl}F_{\text {rep } 1}+F_{\text {rep } 2}, & X-X_{\text {obs }} \leq D \\ 0 & , \text { others }\end{array}\right.$

where,

$$
F_{r e p 1}=K_{r e p}\left(\frac{1}{X-X_{o b s}}-\frac{1}{D}\right) \frac{1}{\left(X-X_{o b s}\right)^{2}} \frac{\partial\left(X-X_{o b s}\right)}{\partial X}\left(X-X_{g}\right)^{n}
$$

$F_{r e p 2}=-\frac{1}{2} K_{r e p}\left(\frac{1}{X-X_{o b s}}-\frac{1}{D}\right)^{2} \frac{1}{\left(X-X_{o b s}\right)^{2}} \frac{\partial\left(X-X_{g}\right)^{2}}{\partial X}$

The obstacle near the target point has no repulsion to the automated guided vehicle which approaches the target point, now $F_{\text {rep } 1}$ tends to zero, and the automated guided continues to the target point under the driving of $F_{\text {rep } 2}$.

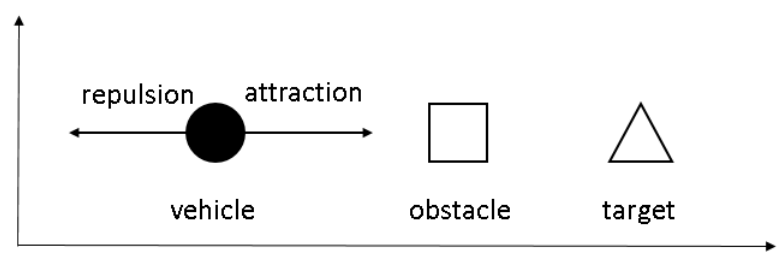

FIGURE III. LOCAL MINIMUM TRAP

In addition, the artificial potential field also has the problem of local minimum trap. When the target, obstacles and automated guided vehicle in the same line, as shown in Figure 3, the force of vehicle is zero, but the vehicle did not reach the target point at this time. In this paper, the method of adding additional force is adopted to solve the problem. When the automated guided vehicle detects that the resultant force is zero, an additional force that is perpendicular to the gravitational direction is added to cause it to escape from the local minimum trap. 


\section{THE IMPLEMENTATION STEPS OF HYBRID PATH PLANNING ALGORITHM}

It is difficult for the unmanned vehicle to rely on the traditional single path planning algorithm to accomplish the task under the premise of ensuring high efficiency and safety in the storage system. Therefore, this paper proposes a new hybrid algorithm that combines the improved $\mathrm{A}^{*}$ algorithm with the improved APF for path planning, which can effectively avoid the obstacles while planning the global optimal path. The steps are as below.

Firstly, the global optimal path is planned by the improved A* algorithm, then the automated guided vehicle tracks the path. In the process of path tracking, random obstacles on the path are detected in real time. The obstacle boundary and relative position information are generated according to the detection results, and the road environment is judged based on the results. Finally, the global path planning and the local path planning algorithm are switched according to the judgment results.

Method for judging road environment as below. Firstly, calculate the distance between the edge of a barrier and the wall. Then determine whether the distance is less than the safe distance that automated guided vehicle can pass. If the distance is less than the safe distance, update the adjacency matrix and restart the global path planning with the current location as the starting point. If the distance is greater than the safe distance, then the improved APF is switched to avoid the obstacle. The flow chart of the hybrid algorithm is shown in Figure 4.

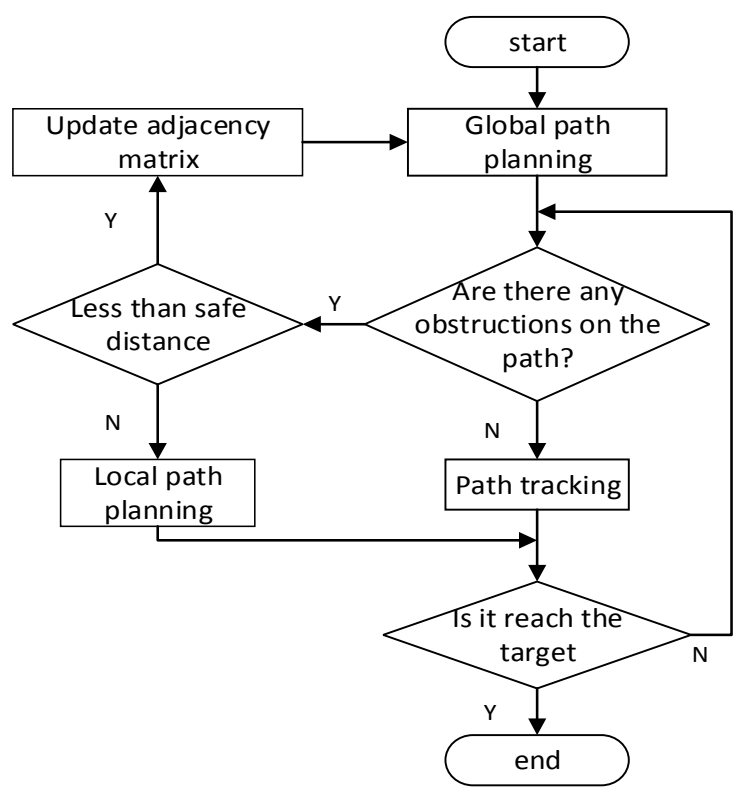

FIGURE IV. FLOW CHART OF HYBRID ALGORITHM

\section{SIMULATION AND EXPERIMENTAL RESULTS}

\section{A. Simulation results of Global Path Planning}

The global path planning adopts the improved A* algorithm to compute the global optimal target point. The path planning results of automated guided vehicles at different starting positions are shown in Figure 5. It can be seen that when the automated guided vehicle is in different initial position, the global path planning algorithm can complete the path planning task from the starting point to the target point, and the calculated path is the global shortest path. In Figure (a), the target is reached after 12 calculations. In Figure (b), the target is reached after 12 calculations. Compared to grid map, the computation is obviously reduced.

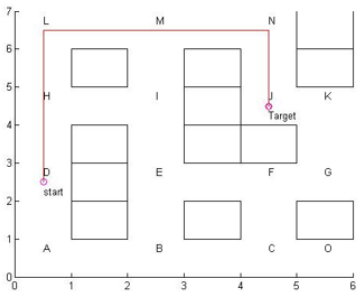

(a)

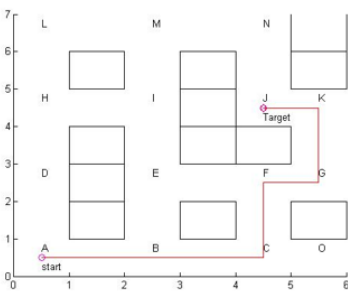

(b)
FIGURE V. SIMULATION RESULTS OF GLOBAL PATH PLANNING

\section{B. Simulation Results of Local Path Planning}

The local path planning algorithm is realized by the improved APF. The simulation result is shown in Figure 6. It can be seen from the simulation results that the vehicle can approach the target gradually while avoiding obstacles. The deflection angle of the vehicle in the local path planning is shown in Figure 7. The path planning method proposed in this paper has a relatively smooth deflection angle and can meet the kinematic constraints of automated guided vehicles.

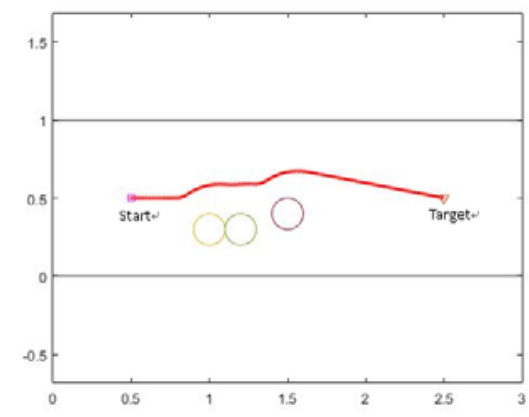

FIGURE VI. SIMULATION RESULTS OF LOCAL PATH PLANNING

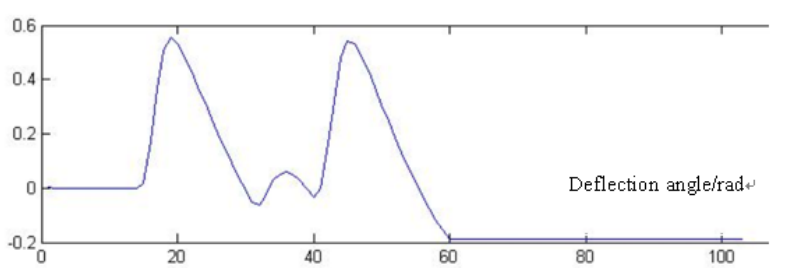

FIGURE VII. DEFLECTION ANGLE OF THE VEHICLE 


\section{Simulation Results of Hybrid Algorithm}

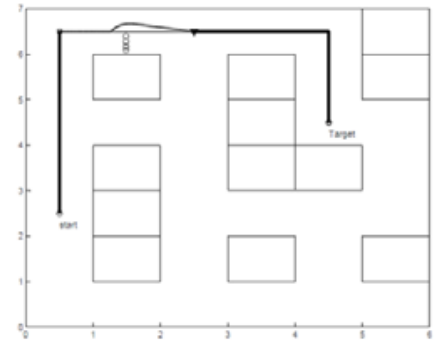

(a)

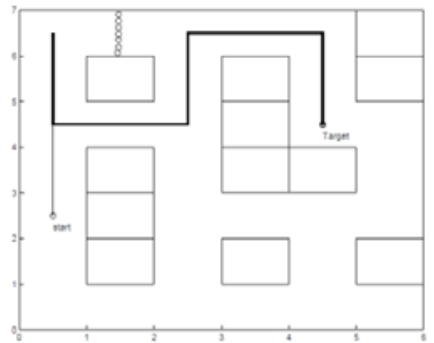

(b)
FIGURE VIII. THE SIMULATION RESULTS OF THE HYBRID PATH PLANNING ALGORITHM

The simulation results of the hybrid path planning algorithm are shown in Figure 8. It can be seen from the Figure (a) that when the automated guided vehicle encounters the obstacle which can be bypassed, it switches to the local path planning algorithm to avoid obstacle, and when the obstacle avoidance is finished, the nodes of the global path are continued to be tracked. The Figure (b) indicates that when an unmanned vehicle encounters an obstacle that cannot be bypassed, the global path planning is started with the current location as the starting point.

The simulation results show that the hybrid algorithm of path planning is proposed in this paper can accomplish the task that reach the target from the current position, and can ensure that the shortest path, no touch throughout.

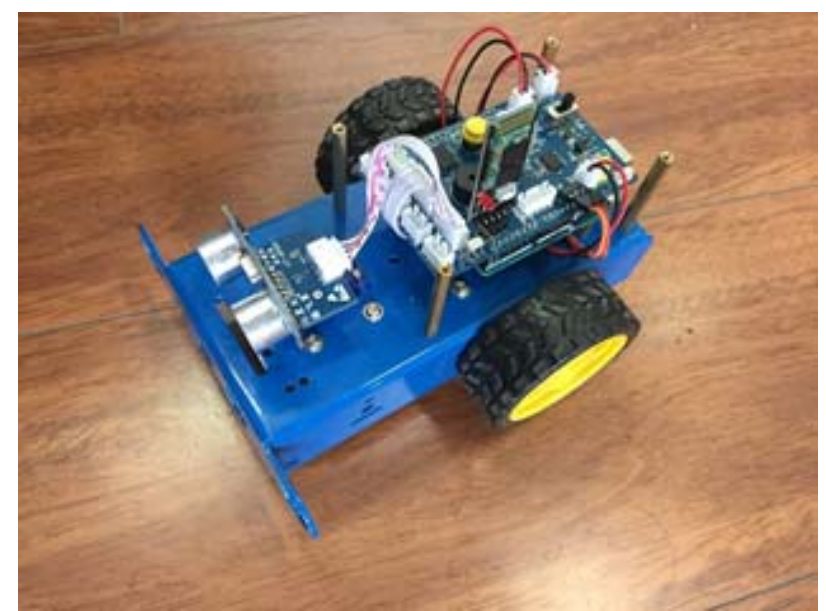

FIGURE IX. THE DOFLYE OPEN SOURCE INTELLIGENT VEHICLE

\section{Experiment}

The hybrid path planning algorithm proposed in this paper has been verified by experiments with the Doflye open source intelligent vehicle. The Doflye open source intelligent vehicle is depicted in Figure 9. This vehicle is driven by two wheel differential drive, and most of the storage vehicles are driven by this type of drive nowadays. The control method of this paper is refs [10]. The experimental results show that the automated guided vehicle can avoid the obstacles while tracking the global path, and finally complete the path tracking task.

\section{CONCLUSION}

Aiming at the problem of automated guided vehicle path planning in storage system, this paper provided a new method which combines global path planning algorithm and local path planning algorithm. The method can effectively avoid obstacles while searching for the optimal path. An improved scheme is put forward according to the large computational complexity of $\mathrm{A}^{*}$ algorithm. At the same time, an improved scheme is proposed for the APF of the target unreachable and the local minimum trap. Finally, the purpose of low cost, high efficiency, safety and no touch is achieved.

\section{ACKNOWLEDGMENT}

The authors thank the home university, Nanjing University of Science \& Technology, for the support.

\section{REFERENCES}

[1] Miyamoto T, Inoue K. Local and random searches for dispatch and conflict-free routing problem of capacitated AGV systems[J]. Computers \& Industrial Engineering, 2015, 91:1-9.

[2] Guo E D, Liu N B, Wu L H, et al. A Path Planning for AGV Based on Genetic Algorithm[J]. Sci-tech Innovation and Productivity, 2016.

[3] Yu J, Lavalle S M. Optimal Multi-Robot Path Planning on Graphs: Structure and Computational Complexity[J]. Computer Science, 2015.

[4] Bell M G H. Hyperstar: A multi-path Astar algorithm for risk averse vehicle navigation[J]. Transportation Research Part B Methodological, 2009, 43(1):97-107.

[5] Zhan W L, Hsu D, Lee W S. Adaptive Informative Path Planning in Metric Spaces[J]. International Journal of Robotics Research, 2015, 35:283-300.

[6] Li C, Jiang X, Wang W, et al. A Simplified Car-following Model Based on the Artificial Potential Field[J]. Procedia Engineering, 2016, 137:1320.

[7] Montiel O, Orozco-Rosas U, Sepúlveda R. Path planning for mobile robots using Bacterial Potential Field for avoiding static and dynamic obstacles[J]. Expert Systems with Applications, 2015, 42(12):5177-5191.

[8] Fazlollahtabar H, Saidimehrabad M. Autonomous Guided Vehicles: Methods and Models for Optimal Path Planning[J]. Optimization, 2015.

[9] Naeem W. Improved APF strategies for dual-arm local motion planning[J]. Transactions of the Institute of Measurement \& Control, 2015, 37:73-90.

[10] Al-Mayyahi A, Wang W, Birch P. Path tracking of autonomous ground vehicle based on fractional order PID controller optimized by PSO[C]// IEEE, International Symposium on Applied Machine Intelligence and Informatics. IEEE, 2015:109-114. 